\title{
Blink Synchronized Brain Activation: Association With Ascending Arousal Network
}

\section{Şükrü Barış Demiral ( $\nabla$ demiralsb@nih.gov)}

National Institute on Alcohol Abuse and Alcoholism, National Institutes of Health https://orcid.org/00000002-5288-603X

\section{Christopher Liu}

National Institute on Alcohol Abuse and Alcoholism, National Institutes of Health

\section{Helene Benveniste}

Yale University

\section{Dardo Tomasi}

National Institute on Alcohol Abuse and Alcoholism, National Institutes of Health

\section{Nora Volkow}

National Institute on Alcohol Abuse and Alcoholism, National Institutes of Health

\section{Article}

Keywords: AAN, VTA, PAG

Posted Date: October 28th, 2020

DOI: https://doi.org/10.21203/rs.3.rs-93881/v1

License: (1) This work is licensed under a Creative Commons Attribution 4.0 International License. Read Full License 


\section{Abstract}

Eye-blinking has been implicated in arousal and attention. Here we test the hypothesis that, blinking-moments represent arousal transitions associated with activation of the ascending arousal network (AAN) and its thalamic projections. For this purpose, we explored the temporal relationship between eye-blinks and fMRI BOLD activity in the AAN and thalamus during a choice-reward task and an eyes-open resting state. We show that during the task, blinks were associated with activation of AAN and thalamic nuclei at the blink moment. During rest, peaks of AAN and thalamic nuclei were not synchronized and appeared weaker except for the ventral tegmental area (VTA). Our findings of an association between eye blinks and activation of the AAN and thalamus provides strong evidence in support of the role of eye blinking in arousal transitions that are influenced by cognitive states. It also corroborates the involvement of the dopaminergic VTA nucleus in eye blinking.

\section{Introduction}

Eye blinks are crucial for eye lubrication and cleansing of the cornea but their complex neuronal modulation including that from the dopaminergic system ${ }^{1-7}$-though see ${ }^{8,9}$ - and other ascending arousal brainstem nuclei also implicate them in arousal and attention functions. Indeed, blinking has been shown to be sensitive to arousal and cognitive states (e.g., attentional allocation, transitions in information flow etc.) ${ }^{10,11}$ and amenable to conscious modification.

It has been suggested that eye blinks might represent a mechanism for dynamic neuro-modulation of brain function ${ }^{12,13}$. Specifically, blinking may serve to help engage arousal regions such as the precuneus 14,15 , which is part of the default mode network (DMN) or task negative network ${ }^{16}$ implicated in self-processing ${ }^{17}$. Indeed, brain imaging data obtained while participants watched movies, showed that blinks coincided with segments of attentional breakpoints and were associated with transient activation of the DMN and deactivation of the dorsal attention network suggesting modulation of internal and external attention orienting networks ${ }^{18}$. Further, the state of consciousness of patients with persistent vegetative and minimally conscious states could be inferred by blink-correlated oscillations in the delta and alpha frequency bands of posterior EEG channels ${ }^{19,20}$. However, the neuronal pathways that underlie blinks and their downstream functional associations including the ascending arousal network (AAN), which is critical to arousal, are poorly understood. The AAN is comprised of brainstem serotonergic (dorsal and medial raphe), noradrenergic (locus coeruleus), glutamatergic (parabrachial complex), dopaminergic (ventral tegmental area) and cholinergic (pontis oralis, pedunculopontine) nuclei and the periaqueductal grey (PAG), which project to the thalamus and cortex 21,22 .

The fMRI derived resting-state networks and functional brain connectivity are believed to reflect brief transitions in spontaneous activity $23-25$, described as point processes where momentary peaks in activity contribute to the variability in functional connectivity 26,27 . Here we hypothesize that blinks would be associated with point-process-like transient peak activations in the AAN and in downstream thalamic regions.

To test this hypothesis, we assessed the dynamic temporal associations between AAN nuclei and thalamus and spontaneous eye-blinks both during rest and task fMRI sessions by monitoring their activity prior to, 
during and post blink. We specifically hypothesized that eye blinks would engage AAN nuclei and the thalamus such that their activity would start to increase prior to the blink and peak during the blink and that this association would be sensitive to the task condition. We selected a choice-reward Task that requires motivation, attention and arousal processes for optimal performance and compared it with an eyes-open Rest condition to conduct a temporal analysis of transient blink synchrony with AAN nuclei ${ }^{22}$ and the thalamus ${ }^{28}$, which integrates AAN signals ${ }^{29}$. Additionally, we performed exploratory brain-wide analyses to identify other potential downstream cortical and subcortical regions that synchronized with blinks. Based on prior findings on blink associated activation of visual regions ${ }^{30-32}$ presumably to sustain uninterrupted visual stream during transient eye closure ${ }^{33}$ and of cerebellar involvement in blink conditioning ${ }^{34}$ we hypothesized blink associated occipital and cerebellar activation. The analyses revealed synchronization of eye blinks with AAN nuclei including VTA and with the thalamus that appeared stronger for the Task than the Rest condition. For both conditions, blinks showed strong but delayed synchronization with cerebellum as well as synchronization with occipital cortex that preceded the blink and continued post blink and that was stronger for Rest than Task condition.

\section{Materials And Methods}

\section{Participants}

Sixteen healthy subjects (8 males, 8 females; ages: 21-63, mean=41.2) completed the fMRI study after providing signed informed consent, approved by the Institutional Review Board at the National Institutes of Health (Combined Neurosciences White Panel). Participants were recruited and screened to exclude ferromagnetic implants, psychoactive medications and major medical problems, substance use disorders (other than nicotine), and neurological or psychiatric disorders (including eating disorders), as assessed by the Structured Clinical Interview for the Diagnostic and Statistical Manual of Mental Disorders (DSM-IV) ${ }^{35}$. Data from two subjects were excluded from final analysis due to their failure to achieve criteria described below.

\section{MRI data acquisition sequences}

MRI data was collected on a 3T Magnetom Prisma scanner (Siemens Medical Solutions USA, Inc., Malvern, PA) using a 32-channel head coil. A standard echo planar imaging (EPI) sequence (sequential interleaved acquisition, repetition time $2.13 \mathrm{~s}$, echo time $30 \mathrm{~ms}$, flip angle $\mathrm{a}=79^{\circ}, 64 \times 64$ pixels in-plane resolution, 40 slices, slice thickness $3.5 \mathrm{~mm}$, voxel dimensions $3 \times 3 \times 3.5 \mathrm{~mm}^{3}$, field of view $192 \times 192 \mathrm{~mm}^{2}$ ) was used for functional scans. The three-dimensional, magnetization-prepared rapid gradient-echo ${ }^{36}$ (3D MP-RAGE; TR/TE = 2400/2.24ms, FA = $8 \mathrm{deg}, \mathrm{TI}=1060 \mathrm{~ms}$ ), and Sampling Perfection with Application optimized Contrasts by using different flip angle Evolutions (SPACE, Siemens; TR/TE $=3200 / 564 \mathrm{~ms}$ ) pulse sequences were used to acquire high-resolution anatomical brain images with $0.8 \mathrm{~mm}$ isotropic voxels field-of-view $(\mathrm{FOV})=240 \times 256$ $\mathrm{mm}$, matrix $=300 \times 320$, and 208 sagittal slices.

\section{Experimental procedures}

All participants underwent two fMRI scan sessions, each consisting of three runs ( 13 minutes each) of a choice-reward task and two resting state runs ( 10 minutes each, prior to and after the choice-reward fMRI 
task). Both choice-reward task and resting state experiments were developed using E-Prime software (Psychology Software Tools, Pittsburgh, PA, version 2.0.10 Professional). Stimuli were presented on a black background under dimmed room lighting using a liquid-crystal display screen (BOLDscreen 32, Cambridge Research Systems; UK). During the experiment the E-Prime computer communicated with the eye-tracker and the fMRI computers, either to send stimulus onset triggers, or to receive stimulus to start and stop the experiment. During the resting scan subjects were asked to keep their eyes open and to stare at a white cross on a black background and minimize eye movements. The choice-reward task is described in detail in Supplementary Methods. Briefly, participants were given $10 \$$ worth of points to start the game. The reward value of the trial (low, 5 or 25 cents; high, 1 or 2 dollars, or Null: 0 money) was shown, and after three seconds, they had to make a choice between two shapes (a triangle or a square) presented side by side separated by a color (red or a green) circle at the center of the field of view. The green circle corresponded to a "GO" trial for which the participant had to make a choice between shapes by pressing the right or left button within a 2seconds response window and their reaction times were measured to assess their association with blink latency. The red circle corresponded to a "NOGO" trial for which participants had been instructed not to respond. Four to six seconds after their response (or no response), a feedback message informed them of their choice outcome (win or lose money, or no gain/loss). For both, GO and NOGO trials winning was preset at $55 \%$ and loosing at $45 \%$, independent of the participant's choices, such that all participants would earn the same amount of points at the end of the task unless they missed a response or made a response to NOGO, situations where they were punished by subtracting 25 cents from their points.

\section{Eye tracking data acquisition and analysis}

During the fMRI scans, we measured pupil diameter and eye-blinks with an ASL (Applied Science Laboratories, previously known as Argus Science Inc., Bedford, MA) long-range LRO eye-tracker camera and ET7 software system. Eye-calibration was performed before the resting and task scans, and pupil size was normalized for each individual and scaled to the machine units. Stimulus images were recorded along with the eye image, and simultaneous pupil detection and eye-localization were handled automatically by the ET7 software. Sampling rate was set to $120 \mathrm{~Hz}$. To detect eye-blinks, we used ET Analysis (version 1.0.4.4) software's default settings where blink duration was set to a minimum of $0.1 \mathrm{~s}$ and a maximum of $0.4 \mathrm{~s}$, and to a minimum pupil diameter of 25 units. Temporary pupil disappearances within these values were recorded as "blinks". Any other interval of data where the pupil was not detected was classified as "signal loss". We simultaneously registered stimulus event onset times. We tested the reliability of the software performance by eye-balling the pupil size/blink moments and the eye-videos provided by the ASL software on ten randomly chosen blocks from five subjects. For all the subjects we also checked the reliability of the pupil detection algorithm and excluded subjects with major problems as discussed below. The software output accurately reflected the manually recorded blinks.

\section{Blink-rate calculation}

For all scans, we calculated blink rates estimated as the number of blinks per number of seconds that the eyes were open per each scan run. We standardize this measure across subjects and used it as a covariate in the SPM model.

\section{Task Related Blink Analysis}


For the choice-reward task, we calculated the blink densities (blink related eye-closures divided by the number of samples) around the response and feedback stimuli between -3 to +4 seconds. We also calculated the correlations between response times and immediate blink onset latencies after Go stimuli.

\section{FMRI analysis}

Using $\mathrm{AFNI}^{37}$, timing differences in slice acquisition were corrected, and each volume was aligned to the first volume using rigid body translations and rotations. To remove physiological and motion-related artifacts, a dual-mask spatial independent component (SIC) analysis was applied to the motion and slice-time corrected functional data for each subject ${ }^{38}$. The resulting components were classified by a machine learning algorithm based on a simple set of spatial features (including out-of-brain ratio, scattering degree etc.). Brain activity was reconstructed by projecting the selected sICs back to the brain space. The results of the algorithm were monitored and checked by a human expert. The functional and anatomical images of each participant were co-registered and normalized to the stereotaxic space of the Montreal Neurological Institute (MNI) using SPM12. The functional images were resampled to a voxel size of $3 \times 3 \times 3 \mathrm{~mm}$ and smoothed with an isotropic 6-mm FWHM Gaussian kernel. Block-level variance fluctuations were removed in a separate SPM model. Finally, low-frequency $(<1 / 128 \mathrm{~Hz})$ scanner drifts were filtered with a high-pass filter. The resulting residual activity was then used for cross-correlation analysis with the blink regressors extracted as described below.

\section{Blink related brain activation analysis}

For each scan series, blink activity extracted from the eye-tracking analysis was convolved with the canonical Hemodynamic Response Function (HRF) in SPM12 (2-gamma function), and then normalized (z-scored). These time series were used for cross-correlation analysis with voxel-wise brain activity in temporal TR (2.13s) lags from -3 to +3 TRs, and the correlations were then transformed to Fisher's z-values.

\section{Data and participant exclusion criteria}

Due to the experiment's complexity (i.e., setting up eye-tracker in an fMRI environment), we expected some well-known problems to emerge such as i) technical problems in eye-tracking quality (i.e., calibration problems, distortions in pupil detection), and ii) non-compliant participants who did not perform the task properly or closed their eyes too often (particularly during the resting scan). Thus, we excluded any runs/sessions where the task and rest eye data had technical problems, such as long eye-closures (i.e., eye closures $>40 \%$ of the time) and weak corneal contrasts that interfered with blink detection. This resulted in exclusion of two participants, and one session from each of four participants. Thus, for the final analysis, we had twenty-four sessions collected from fourteen participants with a total of seventy-two task runs and forty-one resting runs available.

\section{Regions of Interest (ROI) Analyses in the Ascending Arousal Network (AAN) and Thalamus}

We computed correlation activity plots for regions of interest ROI extracted from nine brainstem nuclei from the AAN (https://www.nmr.mgh.harvard.edu/resources/aan-atlas) using $1 \mathrm{~mm}$, MNI152 template ${ }^{22,39}$, that comprised the dorsal raphe (DR), median raphe (MR), median raphe frontalis (MRF), locus coeruleus (LC), 
parabrachial complex (PBC), pontis oralis (PO), periaqueductal grey (PAG), pedunculopontine nucleus (PPN) and ventral tegmental area (VTA). AAN masks are shown in Supplementary Methods. We averaged the zvalues for the serotonergic nuclei (MRF, MR, DR) and for the cholinergic nuclei (PO, PPN) and computed separate values for VTA (dopaminergic), LC (noradrenergic), PBC (glutamatergic) and PAG ${ }^{40}$. We extracted an additional spherical ROI (3mm diameter) centered at MNI coordinates xyz of $0,-15,-12$, which has been used as the location of the VTA ROI by some studies ${ }^{41}$. Thus, we wanted to compare findings in VTA when using coordinates from the AAN atlas versus this ROI location, which is slightly anterior and superior to the AAN ROI. For the thalamus we extracted 7 ROIs from the atlas defined by Behrens et al. ${ }^{28,42}$ which are found to show exclusive white matter connectivity probabilities with major cortical areas (i.e. motor, premotor, frontal, occipital, temporal, parietal and sensory cortices; masks are shown in Supplementary Methods).

\section{Whole Brain Analyses}

For whole brain analyses of blinks associations with brain activity we used SPM12 paired t-test, using the averaged correlation coefficients per subject $(\mathrm{N}=14)$ for Task and Rest conditions and included blink rate as covariate (leading to $\mathrm{df}=12$ ). Normalized (i.e., Fisher's z-transform) correlations (between BOLD and blink regressor) values of all available resting and task blocks were used in the statistical analysis separately for each lag.

We first conducted a cluster-uncorrected analysis for visualization purposes in brain stem regions (voxel tthreshold $t>1.8, k>1,1 \mathrm{~mm}$ isotropic, $\mathrm{p}<.05$, liberal approach). In the second analysis we conducted traditional FWER corrected cluster-based analysis ( $3 \mathrm{~mm}$ isotropic images) to identify significant clusters across large brain areas. The resolution element size, i.e., 'resel', of the images were minimum of 22 voxels leading to around 2985 resels in a group mask, and FWHM was $8.3 \mathrm{~mm}, 8.4 \mathrm{~mm}$, and $8.5 \mathrm{~mm}$ with voxel height

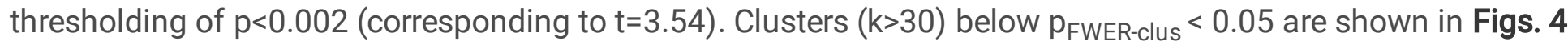
and $\mathbf{5}$ and summarized in Table 1. Detailed cluster maps are given in Supplementary Data 1, 2 and $\mathbf{3}$ and detailed table of results is given in Supplementary Data 4.

\section{Cerebellar ROI Analyses}

Because of the extensive blink associated cerebellar activation signals detected by the whole brain analyses we also extracted 17 ROls from the cerebellum using the functional connectivity atlas defined by Buckner et al. ${ }^{43}$, which possess distinct functional connectivity patterns with cortical subregions (See also ${ }^{44}$ ). To simplify our analysis we averaged the values from most of these ROls to represent their connectivity to 7 main networks defined by the spatial proximity of the target cortical areas (i.e., fronto-parietal attention, DMN, anterior cingulate cortex (ACC), motor, insula, basal forebrain, visual networks). Details of the subregion grouping are given in Supplementary Table 1.

\section{Results}

\section{Behavioral responses: Blink Activity During Task}

Blinks were suppressed before and peaked shortly after signaling of condition (GO vs NOGO) for both Null and Gain trials (Fig 1; see Supplementary Data 5 for feedback related blink changes). Reaction times (Null= 
$.767 \mathrm{~ms}$, sd=.167ms; Gain=.652ms, sd=.145ms) were $115 \mathrm{~ms}$ (s.e. $=53 \mathrm{~ms}$ ) faster for Gain than Null conditions $(t=2.14, p<.05)$. Peak blink density latencies calculated between 0.5 s to 2 s post-stimulus were significantly delayed for GO than NOGO (GO; mean=1205ms, sd=336ms, NOGO; mean=877ms, sd=362ms, F= 30.31, $\mathrm{p}<.001)$. Peak amplitudes did not differ between NULL and GAIN conditions. There was a significant correlation between blink latency and reaction time for the Gain ( $r=0.58, p<.05)$, but not the Null $(r=.05, n s)$ in GO conditions, indicating a strong blink-RT coupling for reinforced trials. In addition, the intercept of a linear model where reaction time was introduced as an outcome variable and the blink latency introduced as a continuous variable revealed that blinks followed button presses on average $342 \mathrm{~ms}$ (s.e. $=112 \mathrm{~ms}$ ) for Gain and $734 \mathrm{~ms}$ (s.e. $=174 \mathrm{~ms}$ ) for Null conditions.

\section{AAN ROI Analysis}

The ROI analyses revealed blink associations with activation of AAN nuclei for the Task and Rest conditions (Fig 2). For the Task condition, the AAN nuclei showed a temporal association with blinks that was strongest in serotonergic (MR/MR/DR) and cholinergic (PO/PPN) nuclei and in LC (norepinephrinergic) and intermediate for VTA (dopaminergic) and PBC (glutamatergic). Analyses of the temporal correspondence of blink synchrony showed that most AAN nuclei peaked at the blink moment except for LC, which peaked post blink.

For the Rest condition the temporal association with blinks appeared weaker (except for VTA) and less coordinated than for the Task condition and most of their peaks occurred earlier than for the Task condition. The cholinergic (PO/PPN) and serotonergic (MR/MR/DR) nuclei and the PBC and LC peaked prior to the blink. Only the VTA, which peaked post blink was delayed compared to the Task.

The results in the VTA for Task and Rest were similar when we computed them using the ROI with MNI coordinates of $0,-15,-12$ to when we computed them with the ROI from the AAN atlas (Supplementary Data 6) indicating that the findings were not sensitive to slight variations in the ROI location.

To estimate if there were differences in the size of the areas showing blink associated activation between Task and Rest conditions we estimated the number of voxels $\left(1 \mathrm{~mm}^{3}\right)$ with greater than $t>1.8$ threshold. Comparison revealed that areas with blink-associated activation allocated significantly larger number of voxels for Task than Rest in all the AAN nuclei at blink moment $\left(\chi^{2}(1, N=9004)=887.9, p<.0001\right)$ except for a marginal effect on the glutaminergic PBC $\left(\chi^{2}(1, N=1460)=3.84, p=.050\right)$.

\section{Thalamic Atlas ROI Analysis}

Analysis of blink-related activations in thalamic ROI extracted from the Behrens atlas appeared to show stronger associations for Task than Rest conditions except for the occipital ROI, which was similar for both conditions (Fig 3, see Supplementary Data 7 for individual values and across subject variability). For Task condition there were strong and coordinated associations among the various thalamic nuclei that peaked during the blink similar to that observed for AAN nuclei during the Task. The ROI with the strongest associations corresponded to the frontal thalamic ROI, followed by pre-motor, and temporal thalamic ROls (have higher structural connections with those cortical regions) and the weakest with the sensory ROI. For the Rest condition just as for the Task, the strongest association were for the frontal thalamic ROI followed by, 
temporal, premotor and occipital thalamic ROls and weakest for the sensory ROI, but different from the Task condition their peaks occurred prior to the blink.

We compared the size of the areas in the thalamic nuclei showing blink associated activation between Task and Rest conditions and revealed that areas with blink-associated activation (number of voxels passing $t>1.8$ threshold) were significantly larger for Task than Rest in all of the thalamic nuclei at the blink moment $\left(\chi^{2}(1\right.$, $\mathrm{N}=35840)=5754.8, \mathrm{p}<.0001)$.

\section{Exploratory Whole Brain fMRI Analysis}

BOLD responses synchronized with blinks for pre-blink, blink and post-blink moments are shown in Fig 4 (for Task), and Fig 5 (for Rest). Blink associated activations differed in location for Task and Rest condition; whereas for the Task condition they were predominantly located in subcortical regions for the Rest condition they were predominantly located in occipital cortex and precuneus. Similarly blink related deactivation also differed, being more extensive for Rest than Task (Supplementary Data 1 and 2 provides comprehensive cluster-based FWER corrected sagittal cluster maps for -2TR to +2TR blink onset for $t>3.54$, $k>30$; voxel threshold $\mathrm{p}<.002)$. However, the only region that showed significant differences in blink associated activation between Task and Rest conditions was the occipital cortex, which showed significantly stronger positive association with blink for Rest than Task (Rest-Task difference map is given in Supplementary Data 3). The detailed location of all significant clusters for all the analyses is provided in Supplementary Data 4.

\section{Task:}

\section{Positive associations:}

Blink associated activations for the Task included regions in brainstem (midbrain and posterior pons), thalamus, cerebellum and caudate (Fig 4 and Supplementary Data 1 and 4). Midbrain signals emerged around blink and persisted post blink. In thalamus the blink associated activation emerged just prior to the blink, increased during the blink with initial activation in lateral and posterior thalamic clusters that extended and included anterior thalamic cluster and the caudate region post-blink (Supplementary Data 1). PCC activation, which emerged just prior to blink did not survive cluster correction. Calcarine sulcus (V1) was active posteriorly during and after blink (Supplementary Data $\mathbf{8}$ shows a flattened map of the visual cortex showing blink associated activations).

In cerebellum (superior middle and lateral) activation signals emerged in a small midline region that enlarged during blink with persistent and strongest activation after blink (Fig 6 and Supplementary Data 1). In the caudate the activation was strongest post-blink.

\section{Negative associations:}

Blink associated deactivations (negative associations) for the Task comprised small clusters in medial ventral prefrontal region, amygdala and parahippocampus that emerged during blink. Blink associated deactivations in the inferior frontal gyrus (IFG), superior temporal gyrus (STG), precentral eye-fields and precuneus emerged during blink expanding post blink (Supplementary Data 1). 
Rest:

\section{Positive associations:}

The strongest activation associated with blinks in the Rest condition occurred in the occipital cortex, precuneus and to a lesser extent the cerebellum and caudate (Fig 5 and Supplementary Data 2). In the occipital cortex the activity was observed beginning prior to the blink and increased during the blink. The regional locations of blink associated activation in the occipital cortex are shown in Supplementary Data 8 displaying a flattened map of the visual cortex contrasting Rest and Task. In the precuneus the activity was observed prior to blink peaking during the blink and decreasing post blink. In the cerebellum (midline anterior ventral region) activity emerged during blink (Fig 6) whereas in caudate activity emerged post blink (Supplementary Data 2).

\section{Negative associations:}

Blink associated deactivation signals were more extensive for Rest than Task in limbic regions (amygdala, parahippocampus), STG, lateral occipital areas and insula emerged in synchrony with the blink and expanded post blink (Supplementary Data 2). Task-negative cortical areas in Task and Rest overlapped with sensorymotor cortex, superior temporal gyrus, and amygdala (Fig 5 and Supplementary Data 2).

\section{Task vs Rest:}

Comparison of activated and deactivated regions associated with the blink (Supplementary Data $\mathbf{3}$ and $\mathbf{4}$ ) showed that Rest had significantly greater number of associations than Task (REST > TASK), mostly corresponding to regions in the occipital cortex prior to and during the blink. No clusters were significantly higher for Task than Rest.

\section{Cerebellar Atlas ROI Analyses}

Analysis of the blink-related activations using ROI extracted from the Buckner cerebellum atlas revealed that they were most prominent in ROls with functional connections with anterior cingulate cortex and with frontoparietal networks for Task and Rest, and for the DMN network in the Rest condition (Fig 6). The ROls connect to motor and visual networks showed the lowest blink associations, which appeared lower for Rest than Task. The peaks occurred post blink both for Task and Rest.

We compared the size of the areas in the cerebellar ROI showing blink associated activation (number of voxels passing $t>1.8$ threshold) between Task and Rest and showed significantly larger areas for Task than Rest in all $\mathrm{ROI}$ around the blink moment $\left(\chi^{2}(1, N=324,736)=16,618.7, \mathrm{p}<.0001\right)$ except in the $\mathrm{DMN}$ region (Rest showing higher number of voxels, $\left.n s, \chi^{2}(1, N=35,360)=2.39, p=.12\right)$, and Visual region where Rest had significantly higher number of voxels than Task $\left(\chi^{2}(1, N=1968)=182.5, p<.0001\right)$.

\section{Discussion}

In this study we document synchronization between blinks and activation signals in AAN, thalamus, cerebellum and occipital cortex and deactivation in several motor and somatosensory cortices and in limbic 
regions. By measuring the temporal synchronicity with spontaneous blinks during Task and Rest conditions we aimed to provide a theoretical framework with which to investigate blink associated activations in the brain during two different mental states. Specifically, as hypothesized, we observed a temporal association between blink activation in AAN nuclei and thalamus, and also with cerebellum and visual cortex the pattern of which differed between the Task and Rest conditions.

For the Task condition the blink associated activation in AAN and thalamus appeared stronger and more coordinated and was significantly larger than for the Rest condition except for the occipital cortex, which showed significantly greater activation for Rest than Task. The blink associated activations in cerebellum were also significantly more extensive for the Task than Rest condition.

\section{Ascending Arousal Network and Thalamus}

Our findings of blink-related associations with AAN nuclei and thalamus corroborated our hypothesis that spontaneous eye blinks would be associated with arousal networks. These findings provide evidence that point processes in the AAN and downstream thalamic regions coincide with blinks and showed that these associations were more prominent during Task than Rest indicating that they are modulated by task conditions.

Because of the evidence linking dopaminergic activity with eye blinks we had hypothesized that the VTA, in particular, would show significant blink-related activation. Our results corroborated this both for VTA ROIs extracted using coordinates from the AAN atlas and those used from fMRI studies of VTA functional connectivity. Thus, our findings further support the involvement of the midbrain dopaminergic system in modulating blinks. Blink associated activation of the VTA was observed both for the Task and Rest conditions though its temporal sequence differed, peaking later for the Rest condition. Eyeblink rates have been proposed as markers of brain dopaminergic activity $5,7,12,45$ and studies have shown that they predicted performance of cognitive tasks that involve working memory, which is modulated by dopaminergic signaling ${ }^{46-49}$. The VTA dopaminergic system is also implicated in motivation, 50,51 which could underlie the reported association between eye blinks and proactive motivation (to achieve a positive outcome) ${ }^{52,53}$ as well as preventive motivation (to prevent a negative outcome) ${ }^{54,55}$.

The LC, which is the main noradrenergic projection to the forebrain is recognized to participate in arousal and wakefulness ${ }^{56}$. The LC modulates pupil size as a function of attention ${ }^{57,58}$. However, the LC's involvement on spontaneous eye blinks to our knowledge has not been reported. There is a dense projection from the LC to the superior colliculus, which coordinates eye and eyelid during blinking and a projection from the trigeminal nerve, that through its supraorbital branch initiates a blink reflex, to the LC ${ }^{59}$. The LC blink associated signal was stronger for the Task than for the Rest condition suggesting that LC's engagement in eye blink might be modulated by attentional process.

We also observed a strong blink associated activation with cholinergic nuclei (pontis oralis and pedunculopontine nucleus) that was stronger for Task than Rest. Both the PO and the PPT are critically involved in the generation of rapid eye movement (REM) sleep and regulate wakefulness ${ }^{60,61}$. To our knowledge there is no evidence of their involvement on eye blink modulation. Of the AAN nuclei we assessed 
the PAG showed the weakest association with Blink for the Task and no association for the Rest. The PAG is implicated in processing of pain and other threats so future studies are needed to assess if such association emerge during exposure to painful or threatening stimuli.

The blink related activation in thalamus was similar to the pattern observed for AAN nuclei including blink related activation that was more extensive for Task than Rest and as for AAN Task condition peaked during the blink. Also, for Task blink related activation was coordinated between the thalamic nuclei (similar temporal pattern), whereas for Rest it was not. The subregions with higher blink activation synchronies were the ones with the strongest functional connections to frontal, pre-motor, and temporal cortices as delineated by the Behrens atlas both for the Task and Rest conditions.

As we interpret our findings, we are unable to determine if the eye blink reflects a behavioral manifestation of the AAN and its thalamic projections or whether the blink triggers the responses in AAN and thalamus. During the Task for some subregions the activation emerges prior to the blink such as for VTA and serotonergic raphe nuclei whereas in LC it follows the blink. The temporal sequence is reversed during the Rest condition for LC, which while weak precedes the blink and the VTA which follows the blink. Thus, it would appear as if the engagement of the various AAN nuclei is influenced by the level of cognitive engagement. By contrasting blink temporal activity in Rest and Task, we aimed to dissociate spontaneous versus contextual arousal related blink behavior. Task synchronized blink activations were observed in most of the AAN nuclei and thalamic regions measured and for most the activation peaked during the blink while for Rest the strongest synchronized activation in AAN was with VTA activity and occurred post blink. Future studies using fMRIs with higher temporal and spatial resolutions will be able to compare blink related temporal activations in AAA and thalamic subregions with greater precision and help clarify further their distinct contributions to arousal. Similarly, the use of PET along with fMRI will help determine the extent to which DA as well as serotonergic and noradrenergic receptors modulate the blink related activation of AAN and thalamus.

\section{Whole brain analysis}

The exploratory whole brain analyses revealed significant blink related activation in cerebellum both for Task and Rest. Cerebellar blink-synchrony emerged later than in AAN and thalamic ROI, indicative of a distinct spatio-temporal alignment than these regions. The Buckner atlas identified that cerebellar regions sensitive to blinks were in ROls with functional connectivity to anterior cingulate cortex, DMN and the fronto-parietal attention network. Though the cerebellum's involvement with arousal has been recently recognized ${ }^{62}$ most studies linking the cerebellum with eye blink have pertained to its role in eye blink conditioning or motor regulation ${ }^{63}$. The extent to which cerebellar activation post blink contributes to arousal requires further investigation.

The whole brain analysis also revealed significant blink associated activation in occipital cortex predominantly for the Rest condition that corroborates prior $\mathrm{fMRI}$ findings showing spontaneous blink associated activation in the primary visual cortex ${ }^{33,64}$. The occipital cortex was the only region for which blink associated activation was significantly stronger for Rest than Task conditions. In the Task there was a distinct area of deactivation in the parieto-occipital fissure. This area is around V3A, which might be related to peripheral visual fields ${ }^{65}$, 
and it is possible that during the Task participants focused on foveal locations to receive for instance Go/NoGo command and to monitor outcomes.

We also observed extensive synchronized blink deactivation in several motor and somatosensory cortical and limbic regions that emerged during the blink for the Rest condition and that were much more constrained for the Task condition. In the Task condition there was a number of small areas of blink related deactivation in ventromedial prefrontal cortex (anterior hub of DMN) and in regions from the Salience Network. We interpret this to reflect blink related activation of arousal networks that contribute to DMN inhibition during the Task.

Our findings from the whole brain analyses of blink related activation and deactivation is opposite to that reported during microsleeps, which showed activation of motor, insular, and parahippocampal regions and deactivations in visual cortex, cerebellum and thalamus when participants dosed off and lost awareness (i.e., microsleeps) ${ }^{66}$. These opposite patterns of results suggest that blinks play a role in the momentary activation of arousal systems, increasing the alertness of the participants to keep their brain engaged with the current environment. Consistent with this interpretation are the reports of increases in blink rates at the end of prolonged periods of wakefulness ${ }^{67}$ or following a night of sleep deprivation 68 .

Analyses of the blink distribution during the Task showed that blinks followed responses during the reward Task, in line with previous reports showing that blinks coincide with salient time points during visual tasks ${ }^{69}$. This behavior probably emerges due to the fact that arousal systems need to be engaged with the task, transiently modulating subcortical and cortical networks in order to improve efficiency of task performance.

\section{Limitations}

In our study we used a 3 Tesla magnet but a 7 Tesla MRI would have given us greater sensitivity to assess activation patterns in small regions as is the case for AAN nuclei ${ }^{70}$. Indeed, our spatial resolution was limited to a voxel size of $3 \mathrm{~mm}$, which can be considered large when imaging very small brainstem nuclei. Our study is also limited by temporal resolution, which made it difficult to properly quantify the temporal sequence of activation patterns linked with eye blinks. The magnet bore is dark and it is unclear how the blink association are affected by different ambient light conditions. Finally, though our sample was small $(n=14)$ we were able to identify distinct patterns or activation and deactivation associated with spontaneous blink activity during Task and Rest conditions.

In summary our findings showing an association of eye blinks with AAN including the VTA and with the thalamus are consistent with the engagement of eye blinks in brain arousal processes. Measuring eye blinks during $\mathrm{fMRI}$ as a regular procedure could be a valuable tool to track arousal levels and potential confounds. Future studies combining high resolution fMRI contrasting resting state blink activity with tasks tapping arousal systems will clarify the temporal dynamics of spontaneous blink activity with the various AAN nuclei.

Reporting summary. Further information on research design is available in the Nature Research Reporting Summary linked to this article.

Data availability The datasets generated or analyzed during the current study are available from the first or corresponding author on reasonable request. 
Code availability The scripts generated and used in the current study are available from the first or corresponding author on reasonable request.

\section{References}

1. Elsworth, J. D. et al. D1 and D2 dopamine receptors independently regulate spontaneous blink rate in the vervet monkey. J Pharmacol Exp Ther259, 595-600 (1991).

2. Jutkiewicz, E. M. \& Bergman, J. Effects of dopamine D1 ligands on eye blinking in monkeys: efficacy, antagonism, and D1/D2 interactions. J Pharmacol Exp Ther311, 1008-1015, doi:10.1124/jpet.104.071092 (2004).

3. Kaminer, J., Powers, A. S., Horn, K. G., Hui, C. N. \& Evinger, C. Characterizing the Spontaneous Blink Generator: An Animal Model. J Neurosci31, 11256-11267, doi:10.1523/Jneurosci.6218-10.2011 (2011).

4. Karson, C. N. et al. An inverse correlation between spontaneous eye-blink rate and platelet monoamine oxidase activity. Br J Psychiatry142, 43-46, doi:10.1192/bjp.142.1.43 (1983).

5. Kleven, M. S. \& Koek, W. Differential effects of direct and indirect dopamine agonists on eye blink rate in cynomolgus monkeys. J Pharmacol Exp Ther279, 1211-1219 (1996).

6. Lawrence, M. S. \& Redmond, D. E., Jr. MPTP lesions and dopaminergic drugs alter eye blink rate in African green monkeys. Pharmacol Biochem Behav38, 869-874, doi:10.1016/0091-3057(91)90255-z (1991).

7. Taylor, J. R. et al. Spontaneous blink rates correlate with dopamine levels in the caudate nucleus of MPTPtreated monkeys. Exp Neuro/158, 214-220, doi:DOI 10.1006/exnr.1999.7093 (1999).

8. Sescousse, G. et al. Spontaneous eye blink rate and dopamine synthesis capacity: preliminary evidence for an absence of positive correlation. The European journal of neuroscience47, 1081-1086, doi:10.1111/ejn.13895 (2018).

9. Dang, L. C. et al. Spontaneous Eye Blink Rate (EBR) Is Uncorrelated with Dopamine D2 Receptor Availability and Unmodulated by Dopamine Agonism in Healthy Adults. eNeuro4, doi:10.1523/ENEURO.0211-17.2017 (2017).

10. Stern, J. A., Walrath, L. C. \& Goldstein, R. The Endogenous Eyeblink. Psychophysiology21, 22-33, doi:DOI 10.1111/j.1469-8986.1984.tb02312.x (1984).

11. Ponder, E. \& Kennedy, W. P. ON THE ACT OF BLINKING. Quarterly Journal of Experimental Physiology18, 89-110, doi:10.1113/expphysiol.1927.sp000433 (1927).

12. Karson, C. N. Spontaneous eye-blink rates and dopaminergic systems. Brain: a journal of neurology 106 (Pt 3), 643-653 (1983).

13. Karson, C. N., Dykman, R. A. \& Paige, S. R. Blink Rates in Schizophrenia. Schizophrenia Bul/16, 344-354 (1990).

14. Liu, C. C., Ghosh Hajra, S., Cheung, T. P. L., Song, X. \& D'Arcy, R. C. N. Spontaneous Blinks Activate the Precuneus: Characterizing Blink-Related Oscillations Using Magnetoencephalography. Frontiers in human neuroscience11, 489, doi:10.3389/fnhum.2017.00489 (2017).

15. Song, A. H. et al. Pharmacological Modulation of Noradrenergic Arousal Circuitry Disrupts Functional Connectivity of the Locus Ceruleus in Humans. J Neurosci37, 6938-6945, doi:10.1523/JNEUROSCI.044617.2017 (2017). 
16. Fox, M. D. et al. The human brain is intrinsically organized into dynamic, anticorrelated functional networks. Proceedings of the National Academy of Sciences of the United States of America102, 96739678, doi:10.1073/pnas.0504136102 (2005).

17. Raichle, M. E. et al. A default mode of brain function. Proceedings of the National Academy of Sciences of the United States of America98, 676-682, doi:10.1073/pnas.98.2.676 (2001).

18. Nakano, T. Blink-related dynamic switching between internal and external orienting networks while viewing videos. Neurosci Res96, 54-58, doi:10.1016/j.neures.2015.02.010 (2015).

19. Bonfiglio, L. et al. Cortical source of blink-related delta oscillations and their correlation with levels of consciousness. Human brain mapping34, 2178-2189, doi:10.1002/hbm.22056 (2013).

20. Bonfiglio, L. et al. Spectral parameters modulation and source localization of blink-related alpha and lowbeta oscillations differentiate minimally conscious state from vegetative state/unresponsive wakefulness syndrome. PloS one9, e93252, doi:10.1371/journal.pone.0093252 (2014).

21. Steriade, M., McCormick, D. A. \& Sejnowski, T. J. Thalamocortical oscillations in the sleeping and aroused brain. Science262, 679-685, doi:10.1126/science.8235588 (1993).

22. Edlow, B. L. et al. Neuroanatomic connectivity of the human ascending arousal system critical to consciousness and its disorders. Journal of neuropathology and experimental neurology71, 531-546, doi:10.1097/NEN.0b013e3182588293 (2012).

23. Liu, X. et al. Arousal transitions in sleep, wakefulness, and anesthesia are characterized by an orderly sequence of cortical events. Neurolmage116, 222-231, doi:10.1016/j.neuroimage.2015.04.003 (2015).

24. Liu, X. \& Duyn, J. H. Time-varying functional network information extracted from brief instances of spontaneous brain activity. Proceedings of the National Academy of Sciences of the United States of America110, 4392-4397, doi:10.1073/pnas.1216856110 (2013).

25. Han, F., Gu, Y. \& Liu, X. A Neurophysiological Event of Arousal Modulation May Underlie fMRI-EEG Correlations. Front Neurosci13, 823, doi:10.3389/fnins.2019.00823 (2019).

26. Tagliazucchi, E., Balenzuela, P., Fraiman, D. \& Chialvo, D. R. Criticality in large-scale brain FMRI dynamics unveiled by a novel point process analysis. Front Physio/3, 15, doi:10.3389/fphys.2012.00015 (2012).

27. Tagliazucchi, E., von Wegner, F., Morzelewski, A., Brodbeck, V. \& Laufs, H. Dynamic BOLD functional connectivity in humans and its electrophysiological correlates. Frontiers in human neuroscience6, 339, doi:10.3389/fnhum.2012.00339 (2012).

28. Behrens, T. E. et al. Non-invasive mapping of connections between human thalamus and cortex using diffusion imaging. Nature neuroscience6, 750-757, doi:10.1038/nn1075 (2003).

29. Fuller, P. M., Sherman, D., Pedersen, N. P., Saper, C. B. \& Lu, J. Reassessment of the structural basis of the ascending arousal system. The Journal of comparative neurology519, 933-956, doi:10.1002/cne.22559 (2011).

30. Hupe, J. M., Bordier, C. \& Dojat, M. A BOLD signature of eyeblinks in the visual cortex. Neurolmage61, 149-161, doi:10.1016/j.neuroimage.2012.03.001 (2012).

31. Bristow, D., Haynes, J. D., Sylvester, R., Frith, C. D. \& Rees, G. Blinking suppresses the neural response to unchanging retinal stimulation. Current biology: CB15, 1296-1300, doi:10.1016/j.cub.2005.06.025 (2005). 
32. Bristow, D., Frith, C. \& Rees, G. Two distinct neural effects of blinking on human visual processing. Neurolmage27, 136-145, doi:10.1016/j.neuroimage.2005.03.037 (2005).

33. Golan, T. et al. Human intracranial recordings link suppressed transients rather than 'filling-in' to perceptual continuity across blinks. Elife5, doi:ARTN e17243 7554/eLife.17243 (2016).

34. Johansson, F., Hesslow, G. \& Medina, J. F. Mechanisms for motor timing in the cerebellar cortex. Curr Opin Behav Sci8, 53-59, doi:10.1016/j.cobeha.2016.01.013 (2016).

35. American Psychiatric Association. (American Psychiatric Association, Washington, DC, 2000).

36. Mugler, J. P. \& Brookeman, J. R. Three-dimensional magnetization-prepared rapid gradient-echo imaging (3D MP RAGE). Magn Reson Med15, 152-157 (1990).

37. Cox, R. W. AFNI: Software for analysis and visualization of functional magnetic resonance neuroimages. Comput Biomed Res29, 162-173, doi:DOI 10.1006/cbmr.1996.0014 (1996).

38. Xu, Y. et al. Denoising the speaking brain: toward a robust technique for correcting artifact-contaminated fMRI data under severe motion. Neurolmage103, 33-47, doi:10.1016/j.neuroimage.2014.09.013 (2014).

39. Bianciardi, M. et al. Toward an In Vivo Neuroimaging Template of Human Brainstem Nuclei of the Ascending Arousal, Autonomic, and Motor Systems. Brain connectivity5, 597-607, doi:10.1089/brain.2015.0347 (2015).

40. Lu, J., Jhou, T. C. \& Saper, C. B. Identification of wake-active dopaminergic neurons in the ventral periaqueductal gray matter. J Neurosci26, 193-202, doi:10.1523/JNEUROSCI.2244-05.2006 (2006).

41. Tomasi, D. \& Volkow, N. D. Functional connectivity of substantia nigra and ventral tegmental area: maturation during adolescence and effects of ADHD. Cerebral cortex24, 935-944, doi:10.1093/cercor/bhs382 (2014).

42. Demiral, S. B. et al. Methylphenidate's effects on thalamic metabolism and functional connectivity in cannabis abusers and healthy controls. Neuropsychopharmacology: official publication of the American College of Neuropsychopharmacology, doi:10.1038/s41386-018-0287-2 (2018).

43. Buckner, R. L., Krienen, F. M., Castellanos, A., Diaz, J. C. \& Yeo, B. T. The organization of the human cerebellum estimated by intrinsic functional connectivity. J Neurophysio/106, 2322-2345, doi:10.1152/jn.00339.2011 (2011).

44. Diedrichsen, J., King, M., Hernandez-Castillo, C., Sereno, M. \& Ivry, R. B. Universal Transform or Multiple Functionality? Understanding the Contribution of the Human Cerebellum across Task Domains. Neuron102, 918-928, doi:10.1016/j.neuron.2019.04.021 (2019).

45. Blin, O., Masson, G., Azulay, J. P., Fondarai, J. \& Serratrice, G. Apomorphine-Induced Blinking and Yawning in Healthy-Volunteers. Brit J Clin Pharmaco30, 769-773 (1990).

46. Braver, T. S. \& Barch, D. M. A theory of cognitive control, aging cognition, and neuromodulation. Neurosci Biobehav Rev26, 809-817, doi:10.1016/s0149-7634(02)00067-2 (2002).

47. Braver, T. S., Barch, D. M. \& Cohen, J. D. Cognition and control in schizophrenia: a computational model of dopamine and prefrontal function. Biol Psychiatry46, 312-328, doi:10.1016/s0006-3223(99)00116-x (1999).

48. Hazy, T. E., Frank, M. J. \& O'Reilly, R. C. Banishing the homunculus: making working memory work. Neuroscience139, 105-118, doi:10.1016/j.neuroscience.2005.04.067 (2006). 
49. Colzato, L. S., Slagter, H. A., Spape, M. M. \& Hommel, B. Blinks of the eye predict blinks of the mind. Neuropsychologia46, 3179-3183, doi:10.1016/j.neuropsychologia.2008.07.006 (2008).

50. Volkow, N. D. et al. Methylphenidate-elicited dopamine increases in ventral striatum are associated with long-term symptom improvement in adults with attention deficit hyperactivity disorder. J Neurosci32, 841-849, doi:10.1523/JNEUROSCI.4461-11.2012 (2012).

51. Volkow, N. D. et al. Motivation deficit in ADHD is associated with dysfunction of the dopamine reward pathway. Mol Psychiatry16, 1147-1154, doi:10.1038/mp.2010.97 (2011).

52. Barkley-Levenson, E. \& Galvan, A. Eye blink rate predicts reward decisions in adolescents. Dev Sci20, doi:10.1111/desc.12412 (2017).

53. Peckham, A. D. \& Johnson, S. L. Spontaneous Eye-Blink Rate as an Index of Reward Responsivity: Validation and Links to Bipolar Disorder. Clin Psychol Sci4, 451-463, doi:10.1177/2167702615594999 (2016).

54. Byrne, K. A., Norris, D. D. \& Worthy, D. A. Dopamine, depressive symptoms, and decision-making: the relationship between spontaneous eye blink rate and depressive symptoms predicts lowa Gambling Task performance. Cogn Affect Behav Neurosci16, 23-36, doi:10.3758/s13415-015-0377-0 (2016).

55. Cavanagh, J. F., Masters, S. E., Bath, K. \& Frank, M. J. Conflict acts as an implicit cost in reinforcement learning. Nat Commun5, 5394, doi:10.1038/ncomms6394 (2014).

56. Aston-Jones, G. \& Waterhouse, B. Locus coeruleus: From global projection system to adaptive regulation of behavior. Brain Res1645, 75-78, doi:10.1016/j.brainres.2016.03.001 (2016).

57. Dragone, A. et al. Expectancy modulates pupil size during endogenous orienting of spatial attention. Cortex; a journal devoted to the study of the nervous system and behavior102, 57-66, doi:10.1016/j.cortex.2017.09.011 (2018).

58. Samuels, E. R., Hou, R. H., Langley, R. W., Szabadi, E. \& Bradshaw, C. M. Modulation of the acoustic startle response by the level of arousal: comparison of clonidine and modafinil in healthy volunteers.

Neuropsychopharmaco/32, 2405-2421, doi:10.1038/sj.npp.1301363 (2007).

59. Dauvergne, C. et al. Are locus coeruleus neurons involved in blinking? Neurosci Res61, 182-191, doi:10.1016/j.neures.2008.02.005 (2008).

60. Xi, M. C., Morales, F. R. \& Chase, M. H. Interactions between GABAergic and cholinergic processes in the nucleus pontis oralis: neuronal mechanisms controlling active (rapid eye movement) sleep and wakefulness. J Neurosci24, 10670-10678, doi:10.1523/JNEUROSCI.1987-04.2004 (2004).

61. Kroeger, D. et al. Cholinergic, Glutamatergic, and GABAergic Neurons of the Pedunculopontine Tegmental Nucleus Have Distinct Effects on Sleep/Wake Behavior in Mice. J Neurosci37, 1352-1366, doi:10.1523/JNEUROSCI.1405-16.2016 (2017).

62. Styliadis, C., loannides, A. A., Bamidis, P. D. \& Papadelis, C. Distinct cerebellar lobules process arousal, valence and their interaction in parallel following a temporal hierarchy. Neurolmage110, 149-161, doi:10.1016/j.neuroimage.2015.02.006 (2015).

63. Bao, S., Chen, L., Kim, J. J. \& Thompson, R. F. Cerebellar cortical inhibition and classical eyeblink conditioning. Proceedings of the National Academy of Sciences of the United States of America99, 15921597, doi:10.1073/pnas.032655399 (2002). 
64. Gawne, T. J. \& Martin, J. M. Activity of primate V1 cortical neurons during blinks. J Neurophysio/84, 26912694, doi:10.1152/jn.2000.84.5.2691 (2000).

65. Mackey, W. E., Winawer, J. \& Curtis, C. E. Visual field map clusters in human frontoparietal cortex. Elife6, doi:10.7554/eLife.22974 (2017).

66. Poudel, G. R., Innes, C. R., Bones, P. J., Watts, R. \& Jones, R. D. Losing the struggle to stay awake: divergent thalamic and cortical activity during microsleeps. Human brain mapping35, 257-269, doi:10.1002/hbm.22178 (2014).

67. Barbato, G. et al. Increased spontaneous eye blink rate following prolonged wakefulness. Physiology \& behavior90, 151-154, doi:10.1016/j.physbeh.2006.09.023 (2007).

68. Crevits, L., Simons, B. \& Wildenbeest, J. Effect of sleep deprivation on saccades and eyelid blinking. Eur Neuro/50, 176-180, doi:10.1159/000073060 (2003).

69. Siegle, G. J., Ichikawa, N. \& Steinhauer, S. Blink before and after you think: Blinks occur prior to and following cognitive load indexed by pupillary responses. Psychophysiology45, 679-687, doi:10.1111/j.1469-8986.2008.00681.x (2008).

70. Bianciardi, M. et al. In vivo functional connectome of human brainstem nuclei of the ascending arousal, autonomic, and motor systems by high spatial resolution 7-Tesla fMRI. MAGMA29, 451-462, doi:10.1007/s10334-016-0546-3 (2016).

\section{Tables}


Table 1

Summary of the anatomical location of clusters showing significant temporal association with blinks for Task and Rest. Cluster corrected $(\mathrm{k}>30, \mathrm{p}<.002$, pFWER <.05). A comprehensive table with all the clusters and their precise locations is in Supplementary Data 4 and a flattened map of the visual cortex showing blink associated activation for Task and Rest is shown in Supplementary Data 8. Abbreviations: L, left, R, right.

Symbols ${ }^{\circledR}$ Depicting voxels/regions with high t-values $(\mathrm{t}>3.54)$ but not surviving cluster correction.

\begin{tabular}{|c|c|c|c|c|}
\hline & & Before Blink & During Blink & After Blink \\
\hline \multirow[t]{11}{*}{ TASK } & \multirow[t]{4}{*}{ Positive } & \multirow{4}{*}{$\begin{array}{l}\text { Posterior cingulate } \\
\text { cortex }^{\Downarrow}\end{array}$} & Cerebellum & Cerebellum, Vermis \\
\hline & & & Thalamus & Thalamus \\
\hline & & & Pons/Midbrain ${ }^{\rrbracket}$ & Caudate \\
\hline & & & $\begin{array}{l}\text { Calcarine sulcus } \\
\text { posterior/V1* }\end{array}$ & $\begin{array}{l}\text { Calcarine sulcus } \\
\text { posterior/V1* }\end{array}$ \\
\hline & \multirow[t]{7}{*}{ Negative } & \multirow{7}{*}{$\begin{array}{l}\text { Amygdala } \\
\text { /Parahippocampus } \\
\text { Orbitofrontal }\end{array}$} & Precentral/eye-fields & Parieto-occipital fissure(V3A) \\
\hline & & & Superior Temporal Gyrus & Superior Temporal Gyrus \\
\hline & & & Amygdala/Parahippocampus & Amygdala/Parahippocampus \\
\hline & & & Temporal/auditory cortex & Temporal/auditory cortex \\
\hline & & & Precuneus & Precuneus \\
\hline & & & Rectal gyrus & Inferior Frontal Gyrus \\
\hline & & & Inferior Frontal Gyrus & \\
\hline \multirow[t]{10}{*}{ REST } & \multirow[t]{4}{*}{ Positive } & $\begin{array}{l}\text { Calcarine sulcus- } \\
\text { anterior/V1 }\end{array}$ & \multirow{4}{*}{$\begin{array}{l}\text { Calcarine sulcus anterior/V1* } \\
\text { Precuneus } \\
\text { Cerebellum }\end{array}$} & $\begin{array}{l}\text { Calcarine } \\
\text { sulcus/posterior/V1 }\end{array}$ \\
\hline & & \multirow[t]{3}{*}{ Precuneus } & & Precuneus \\
\hline & & & & Cerebellum \\
\hline & & & & Caudate \\
\hline & \multirow[t]{6}{*}{ Negative } & & Insular cortex & Insular Cortex \\
\hline & & & R Superior Temporal Gyrus & R Superior Temporal Gyrus \\
\hline & & & Temporal/Auditory Cortex & Temporal Auditory Cortex \\
\hline & & & R Inferior Parietal Lobe & Right Postcentral gyrus \\
\hline & & & Lateral Occipital (BA 19) & Lateral Occipital (BA 19) \\
\hline & & & Amygdala/parahippocampus & Amygdala/parahippocampus \\
\hline
\end{tabular}



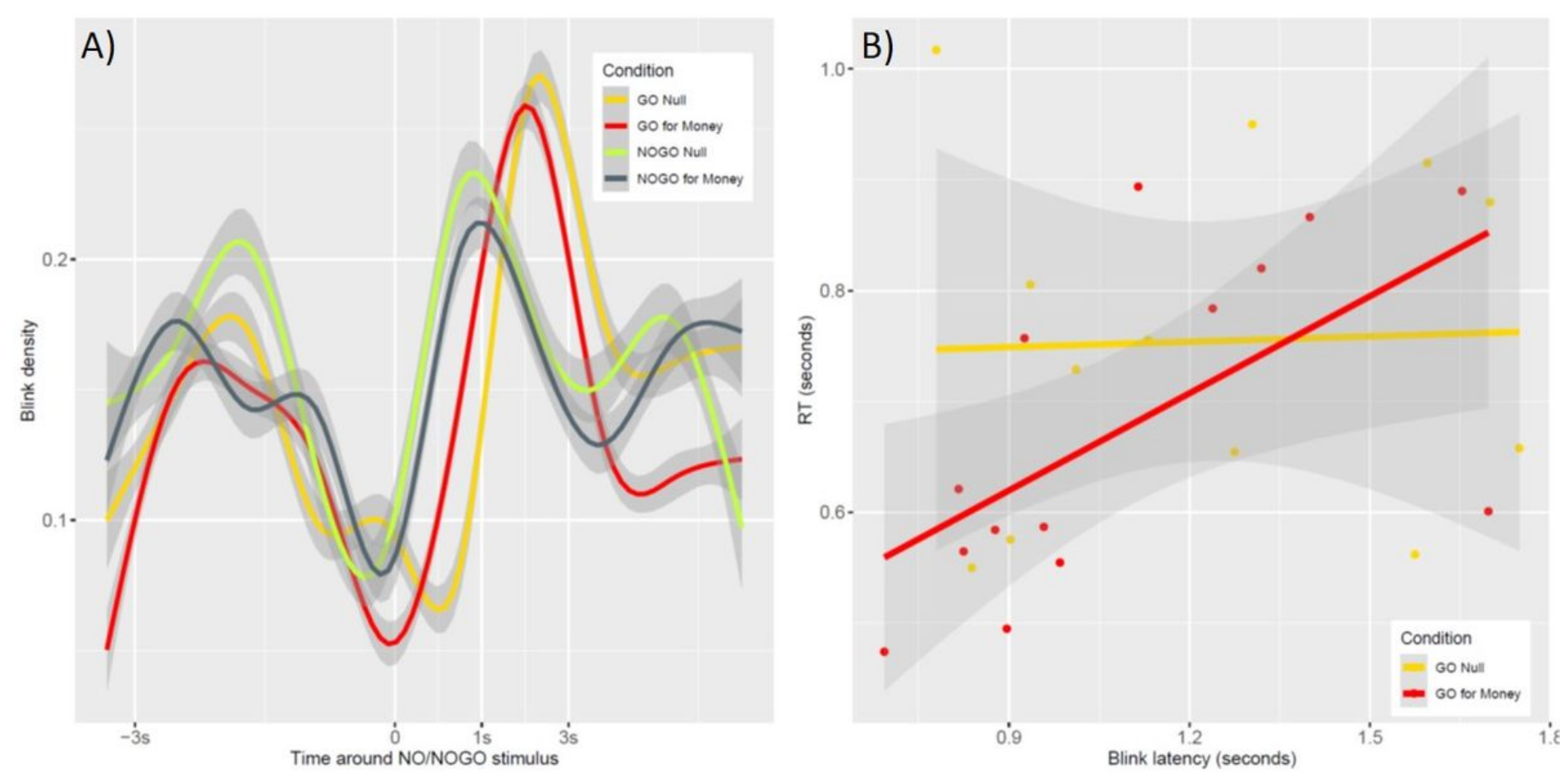

\section{Figure 1}

Blink densities around GO/NOGO stimulus and the relationship between response reaction times and blink latencies for the GO trial. A Blink densities around GO/NOGO stimulus. "Os" is where the GO/NOGO signal was shown. Blink density was lowest around this moment, then increased as time progressed. The peaks for blink densities for GO trials were significantly delayed compared to the NOGO trials. B Correlation between the blink latency upon GO/NOGO signal presentation and reaction time in the response to press the button. Blink latency and reaction time were significantly correlated $(r=0.58, p<.05)$ in $\mathrm{GO}$ incentive trials (for Money) but not in the GO no-incentive trials (Null). 


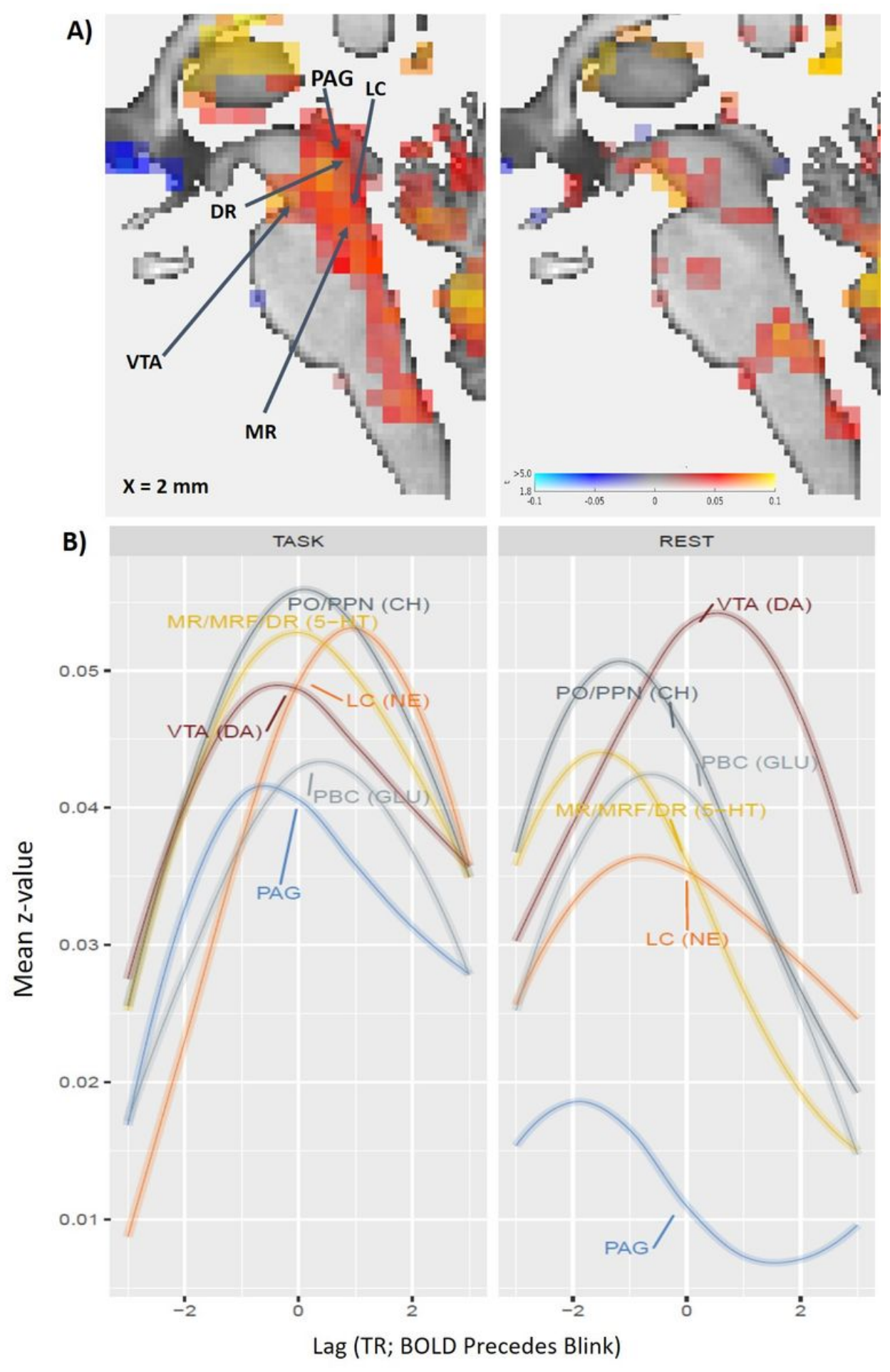

\section{Figure 2}

Blink associations with activation of brainstem AAN nuclei for the Task and Rest conditions. A Sagittal images of midline brainstem ( $\mathrm{MNI} x=2 \mathrm{~mm}$ ) depicting activation in PAG, VTA, LC, MR and DR during the blink. Color scale corresponds to $z$-values across participants and associated t-values (compared to Null distribution). The lower $z$-value was thresholded at $t>1.8$ for visualization purposes. B. Average $z$ values for AAN systems prior to, during blink ( 0 ) and post blink for each temporal lag separately for Task and Rest. Plotting used a 'loess' function to prove a smooth curve. Abbreviations correspond to approximated areas of dorsal raphe (DR), 
median raphe (MR), median raphe frontalis (MRF), locus coeruleus (LC), parabrachial complex (PBC), pontis oralis (PO), periaqueductal grey (PAG), pedunculopontine nucleus (PPN) and ventral tegmental area (VTA).
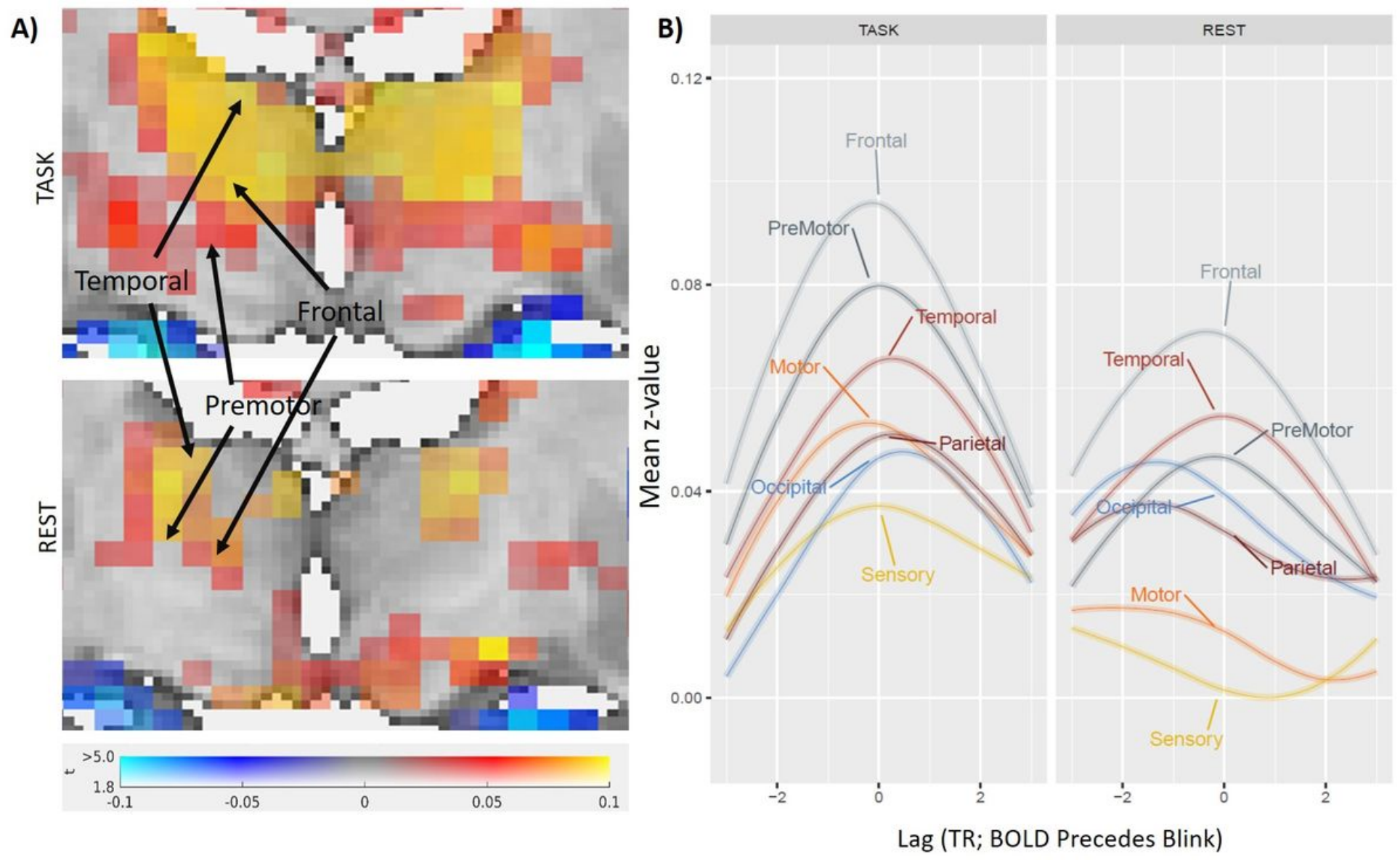

Figure 3

Blink associations with activation of thalamic ROls (Behrens thalamic atlas) for the Task and Rest conditions. A Coronal images of the thalamus ( $\mathrm{MNI}, \mathrm{y}=-13)$ showing the activation during the blink. Color scale corresponds to z-values across participants and associated t-values (compared to Null distribution). The lower $t$-value was thresholded at $t>1.8$ for visualization purposes. The blink associated activation in the thalamus for the Task was widespread and included middle, central and lateral thalamic regions whereas for the Rest condition it was more constrained and mostly limited to lateral-superior thalamic regions. Regions with arrows grossly correspond to Frontal, Temporal and Premotor thalamic segments (see Sup Mat 2 for full subsegments). B Average $z$ values for the thalamic subregion shown for each temporal lag separately for Task and Rest. 


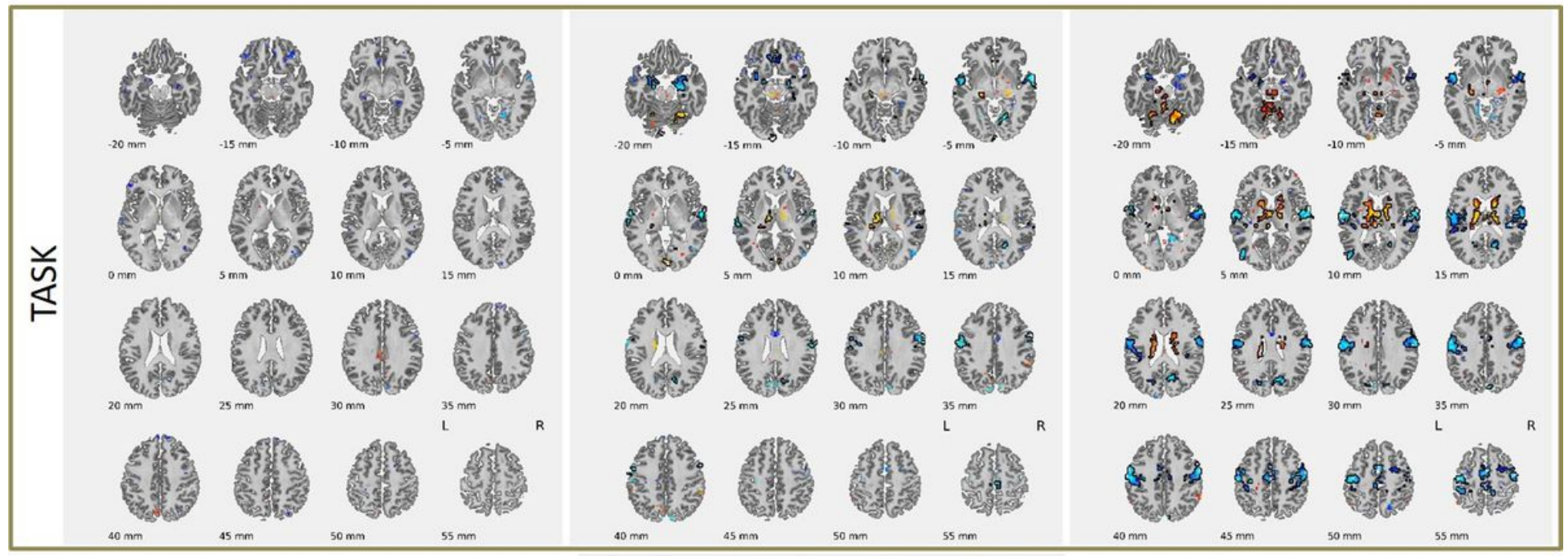

Figure 4

Whole brain cluster analysis for Task. Axial brain images showing blink-associated temporal patterns of activation and deactivation from the whole brain analyses for the Task condition before (-2TR), during, and after (+2TR) blinks. Fisher's z-values are shown for a voxel based statistical threshold $t>3.54$. Surviving clusters with voxel threshold $t>3.54$, one-sample $t$-test, $d f=12, p<.002$, are shown with thick border lines.

BEFORE

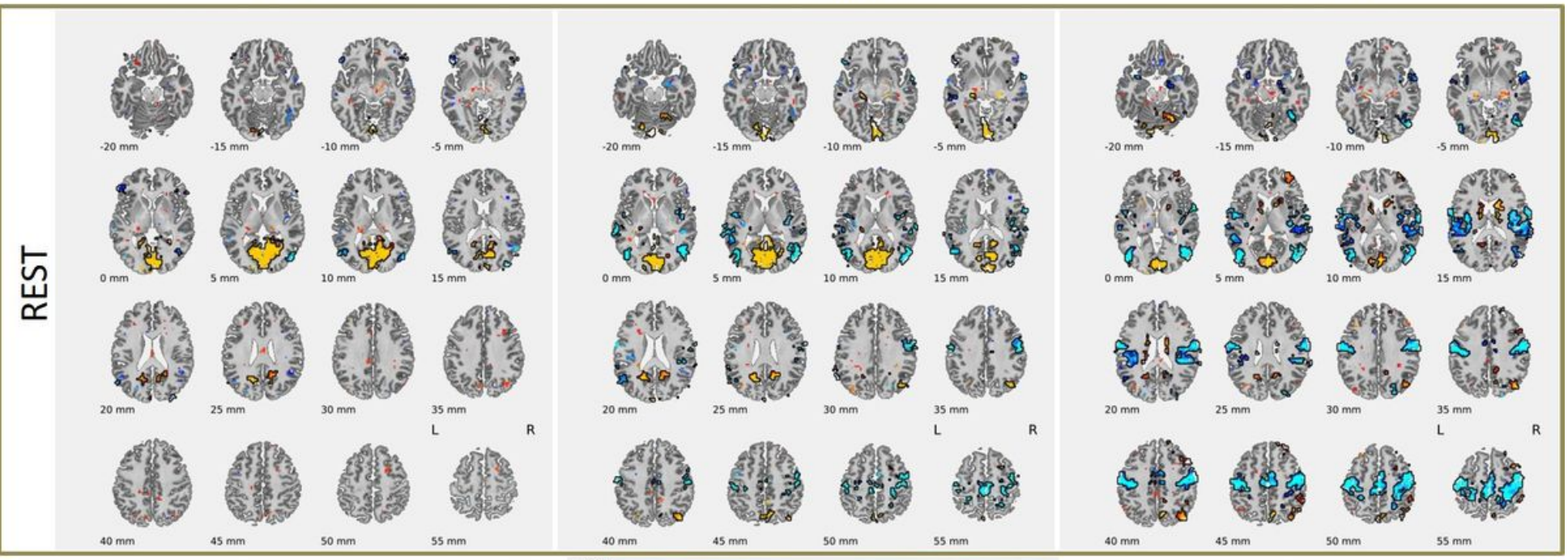

DURING
AFTER

\section{Figure 5}

Whole brain cluster analysis for Rest. Axial brain images showing blink-associated temporal patterns of activation and deactivation from the whole brain analyses for the Rest condition before (-2TR), during, and after (+2TR) blinks. Fisher's z-values are shown for a voxel based statistical threshold $t>3.54$. Surviving clusters with voxel threshold $t>3.54$, one-sample $t$-test, $d f=12, p<.002$, are shown with thick border lines. 
A)
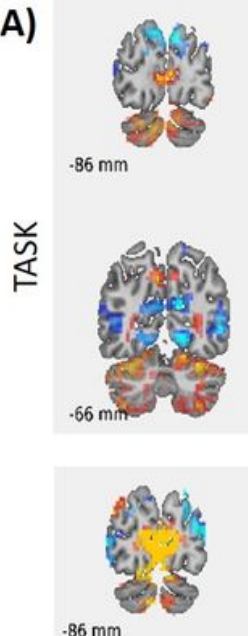

$\stackrel{\llcorner}{\stackrel{\leftarrow}{\sim}}$
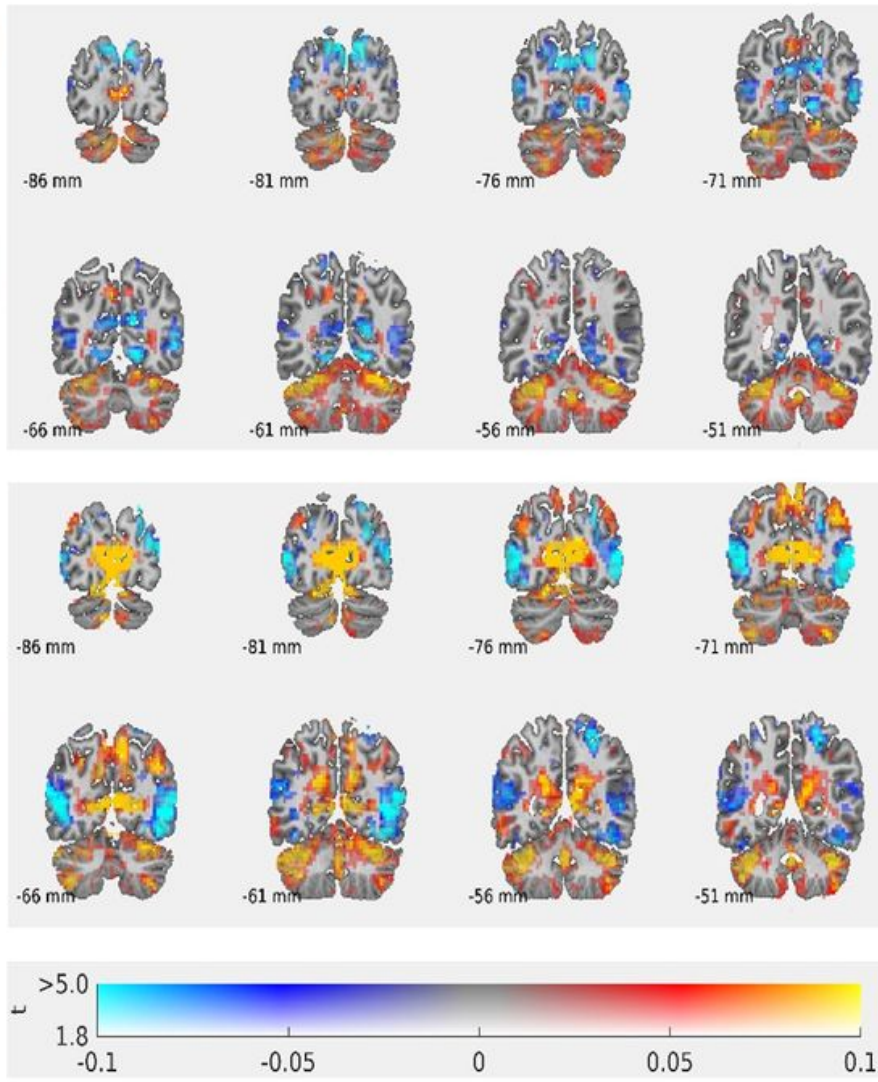

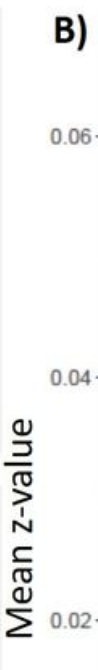

B)

TASK

REST

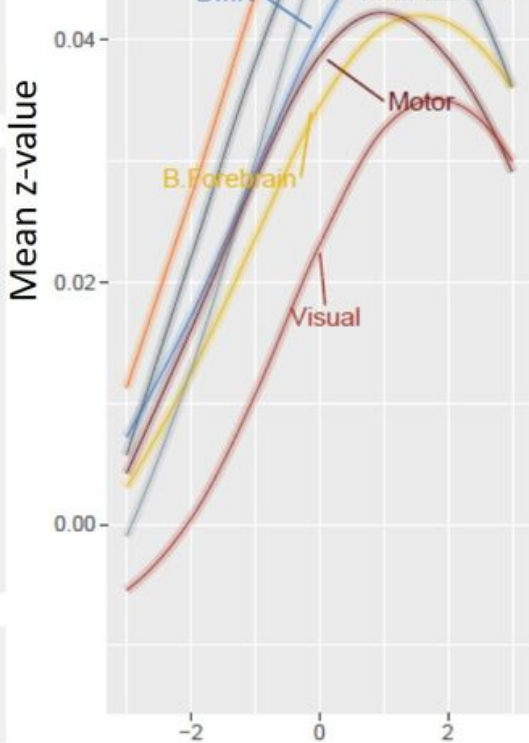

Lag (TR; BOLD Precedes Blink)

\section{Figure 6}

Blink associated activation in Cerebellar ROIs (Buckner cerebellar atlas). A Coronal cerebellar images for Task and Rest conditions. Mean z-values across the participants and corresponding t-values (compared to Null distribution) are shown with the MNI y-coordinates. The lower z-value was thresholded at $t>1.8$ for visualization purposes. B Average $z$ values per cerebellar subregion shown for each temporal lag separately for Task and Rest.

\section{Supplementary Files}

This is a list of supplementary files associated with this preprint. Click to download.

- SupplementaryData8VisualCortexFlattenedMap.docx

- SupplementaryData7InterSubjectVariability.docx

- SupplementaryData6SecondVTAROI.docx

- SupplementaryData5BlinkDensityatFeedback.docx

- SupplementaryData4FWERcorrectedclusterTables.docx

- SupplementaryData3FWERcorrectedclustersRESTTASK.docx

- SupplementaryData2FWERcorrectedclustersREST.docx

- SupplementaryData1FWERcorrectedclustersTASK.docx 
- SupplementaryTable1BucknerCollapseTableupdated.docx

- SupplementaryMethodsTaskDefinitionandBrainMasks.docx

- SupplementaryMethodsTaskDefinitionandBrainMasks.docx

- SupplementaryTable1BucknerCollapseTableupdated.docx

- SupplementaryData1FWERcorrectedclustersTASK.docx

- SupplementaryData2FWERcorrectedclustersREST.docx

- SupplementaryData3FWERcorrectedclustersRESTTASK.docx

- SupplementaryData4FWERcorrectedclusterTables.docx

- SupplementaryData5BlinkDensityatFeedback.docx

- SupplementaryData6SecondVTAROI.docx

- SupplementaryData7InterSubjectVariability.docx

- SupplementaryData8VisualCortexFlattenedMap.docx 